Supporting Information for

\title{
Ultra-incompressible High-Entropy Diborides
}

Xiaoliang Zhang, ${ }^{1}$ Weiwei Li, ${ }^{1}$ Hua Tian, ${ }^{1}$ Junxiu Liu, ${ }^{1}$ Cong Li,${ }^{1}$ Hongliang Dong,,${ }^{1,2}$ Jian Chen, ${ }^{1,3}$ Meng Song, ${ }^{1}$ Bin Chen, ${ }^{1}$ Hongwei Sheng, ${ }^{1}$ Shanmin Wang, ${ }^{3}$ Dongzhou Zhang ${ }^{4}$ and Hengzhong Zhang $^{*}$

1. Center for High Pressure Science and Technology Advanced Research, Shanghai 201203, China

2. State Key Laboratory of High Performance Ceramics and Superfine Microstructure, Shanghai Institute of Ceramics, Chinese Academy of Sciences, Shanghai 201899, China 3. Department of Physics, Southern University of Science and Technology, Shenzhen, Guangdong 518055, China

4. Partnership for Extreme Crystallography Program, University of Hawaii at Manoa, Honolulu, Hawaii 96822, United States 


\section{Sample Syntheses}

The starting materials $\mathrm{TaB}_{2}, \mathrm{NbB}_{2}, \mathrm{ZrB}_{2}, \mathrm{CrB}_{2}, \mathrm{TiB}_{2}$ and $\mathrm{HfB}_{2}$ (purities 99.0 - $99.9 \%$ ) purchased from Aladdin (Shanghai) and/or Alfa Aesar (Haverhill, MA) were used to synthesize the quinary and senary high-entropy diborides. A mixture of the raw materials in the required proportion was ball milled at a high speed (300 - $800 \mathrm{rpm}$ for $\sim 24 \mathrm{~h}$ ) using a planetary ball mill (DECO-PBM-V-0.4L, Changsha). The well homogenized mixture was loaded into a graphite mold $(\phi 20 \mathrm{~mm})$ and then sandwiched by two cylindrical graphite bars, forming a sample with a thickness of $\sim 3-4 \mathrm{~mm}$. The sample assembly was then loaded into the heating chamber of a spark plasma sintering furnace (HP D 10-SI, FCT, Frankenblick). The chamber was evacuated and filled with argon gas, and a static pressure of $\sim 30 \mathrm{MPa}$ was applied to the sample via the two graphite bars. Then, the sample was raised to $2000-2100{ }^{\circ} \mathrm{C}$ at a heating rate of $110{ }^{\circ} \mathrm{C} \min ^{-1}$ and held at the temperature for 15 minutes, followed by a quick cooling to room temperature.

\section{Sample Characterizations}

XRD was used to identify the phases and determine the crystal structures of the prepared HEB samples at ambient pressure using an X-ray diffractometer (Malvern Panalytical) with a $\mathrm{Cu} \mathrm{K} \mathrm{K}_{\alpha}$ radiation (wavelength $1.54056 \AA$ ) operated at $40 \mathrm{kV}$ and $40 \mathrm{~mA}$. The morphologies of the HEB samples were examined using a Versa 3D dual-beam FIB/SEM (FEI) operated at $5.0 \mathrm{keV}$, and their elemental distributions were mapped using 
the energy dispersive spectroscopy of the SEM operated at $15.0 \mathrm{kV}$. The focused ion beam (FIB) facility of the SEM was used to prepare the TEM specimens of the HEBs quenched from $\sim 55 \mathrm{GPa}$. The nanostructures of the specimens were examined using a JEM-2100 TEM (JEOL) operated at $200 \mathrm{kV}$. Contrasts of the SEM/TEM images were enhanced using the program GIMP. ${ }^{1}$ The metal element contents in the HEBs were determined using an energy dispersive X-ray fluorescence spectrometer (SPECTRO XPOS 05) operated at a voltage up to $50 \mathrm{keV}$.

\section{Hardness Determinations}

The Vickers hardness of a HEB sample was measured using a Vickers diamond indenter (FM-810, FUTURE-TECH CORP., Kawasaki) with a loading of 1.0, 2.0, 2.9, 4.9 and $9.8 \mathrm{~N}$ and a holding time of 15 seconds. Multiple indentations were made at different positions of the sample, generating the average hardness of the sample at a given loading.

The nanoindentation hardness and Young's modulus of a HEB sample were measured on a nanoindenter device (G 200, Agilent Technologies Inc., Palo Alto) using a Berkovich indenter operated at a strain rate of $0.05 \mathbf{s}^{-1}$ and an indenting depth ranging from $0-1 \mu \mathrm{m}$. The hardness and Young's modulus measured between 0.1 and $0.2 \mu \mathrm{m}$ were averaged and taken as the values of one measurement, because only at this depth range, the measured values are more intrinsic to the material. ${ }^{2}$ Up to nine measurements were conducted on 
randomly chosen locations of the sample, which then were averaged as the hardness and Young's modulus of the HEB sample.

\section{High-Pressure XRD}

The high-pressure XRD of a HEB sample was conducted at the 13-BM-C synchrotron beamline station of the Advanced Photon Source (APS) with a photon energy of $28.6 \mathrm{keV}$ (X-ray wavelength $0.4335 \AA$ ). A DAC with a diamond culet size of $300 \mu \mathrm{m}$ and a T301 stainless steel gasket was used to generate the required high pressure on the sample. The sample was loaded into the sample chamber of the DAC together with a few small ruby grains as the pressure calibrant. Compressed neon or silicone oil was used as the pressure transmitting medium. The HP-XRD patterns were collected using a MAR165 CCD detector while the sample was in stationary (the quinary HEB) or in a slow rotation within $\pm 5^{\circ}$ around a vertical axis (the senary HEB). The latter case was found beneficial to overcoming preferred orientation of sample particles in HP-XRD. The collected XRD images were converted to the numerical intensity vs $2 \theta$ data using the Dioptas program. ${ }^{3}$ An NIST $660 \mathrm{a} \mathrm{LaB}_{6}$ standard was used as the XRD standard for calibrating the sample-todetector distance and other instrument parameters, including the instrumental peak broadening. 


\section{DFT Calculations}

The programs CASTEP (academic V. 19.11), ${ }^{4}$ CP2K $\left(\right.$ V.4.1) ${ }^{5}$ and Quantum ESPRESSO (QE) (V.6.1) ${ }^{6}$ were used to do geometric optimizations of relevant simple and high-entropy diborides (Table S7) at different pressures $(0,10,30,50 \mathrm{GPa})$. The GGAPBE functionals ${ }^{7}$ were used to evaluate the electron exchange correlations. The CASTEP computations used norm-conserving pseudopotentials (Table S7) to describe the electronion interactions. A k-point mesh (in the Monkhorst-Pack scheme) of $9 \times 9 \times 8$ was applied to a unit cell of relevant simple diborides; only the gamma point was considered for a HEB taking a $4 \times 4 \times 4$ supercell model. The plane wave energy cutoff was $770 \mathrm{eV}$. The $\mathrm{CP} 2 \mathrm{~K}$ computations used a hybrid Gaussian and plane wave formalism and a pseudopotential approximation. The Goedecker-Teter-Hutter pseudopotentials ${ }^{8}$ and the double- $\zeta$ MOLOPT basis sets ${ }^{9}$ were used. An energy cutoff of 400 Ry was used. All diborides were described using supercell models: for simple diborides, a $4 \times 4 \times 4,5 \times 5 \times 2\left(\operatorname{ReB}_{2}\right)$ or $3 \times 4 \times 3$ $\left(\mathrm{OsB}_{2}\right)$ supercell was used; for a HEB, a $4 \times 4 \times 4$ supercell was used. The QE computations used the pseudopotentials taken from the standard solid-state pseudopotential library. ${ }^{10} \mathrm{~A}$ kinetic energy cutoff of 440 Ry was used for the charge density and potential; an energy cutoff of 55 Ry was used for the wavefunctions. A k-point mesh of $4 \times 4 \times 4,5 \times 5 \times 2\left(\operatorname{ReB}_{2}\right)$ or $3 \times 4 \times 3\left(\mathrm{OsB}_{2}\right)$ was used for simple diborides in a unit cell; the gamma point was used for a HEB in a $4 \times 4 \times 4$ supercell. 


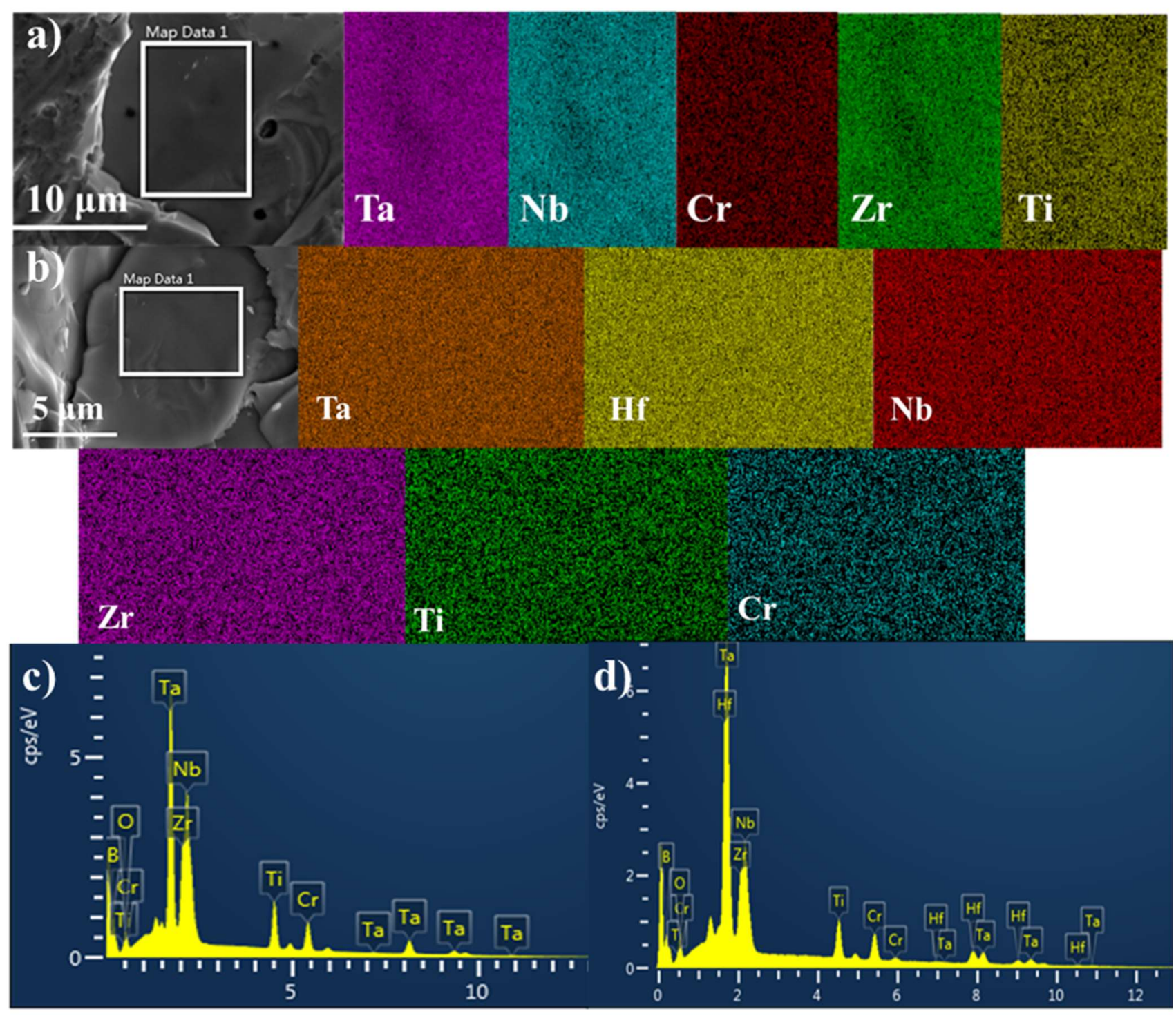

Figure S1. $(a-b)$ EDS mapping showing metal elemental distributions in the quinary (a) and the senary (b) HEBs. (c - d) EDS diagrams of the quinary (c) and the senary (d) HEBs. Trace oxygen was present in the samples in addition to major transition metal elements and boron. 

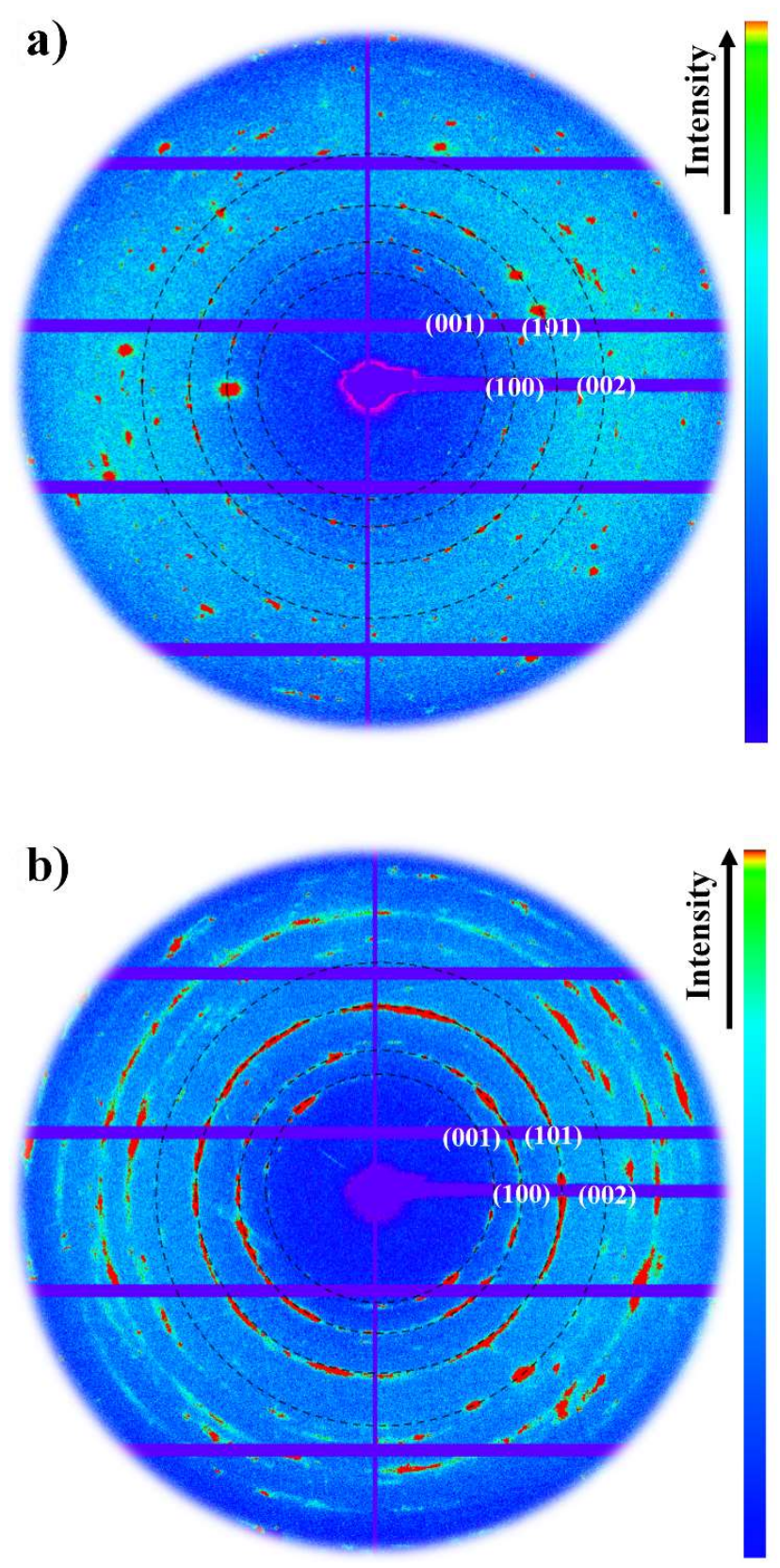

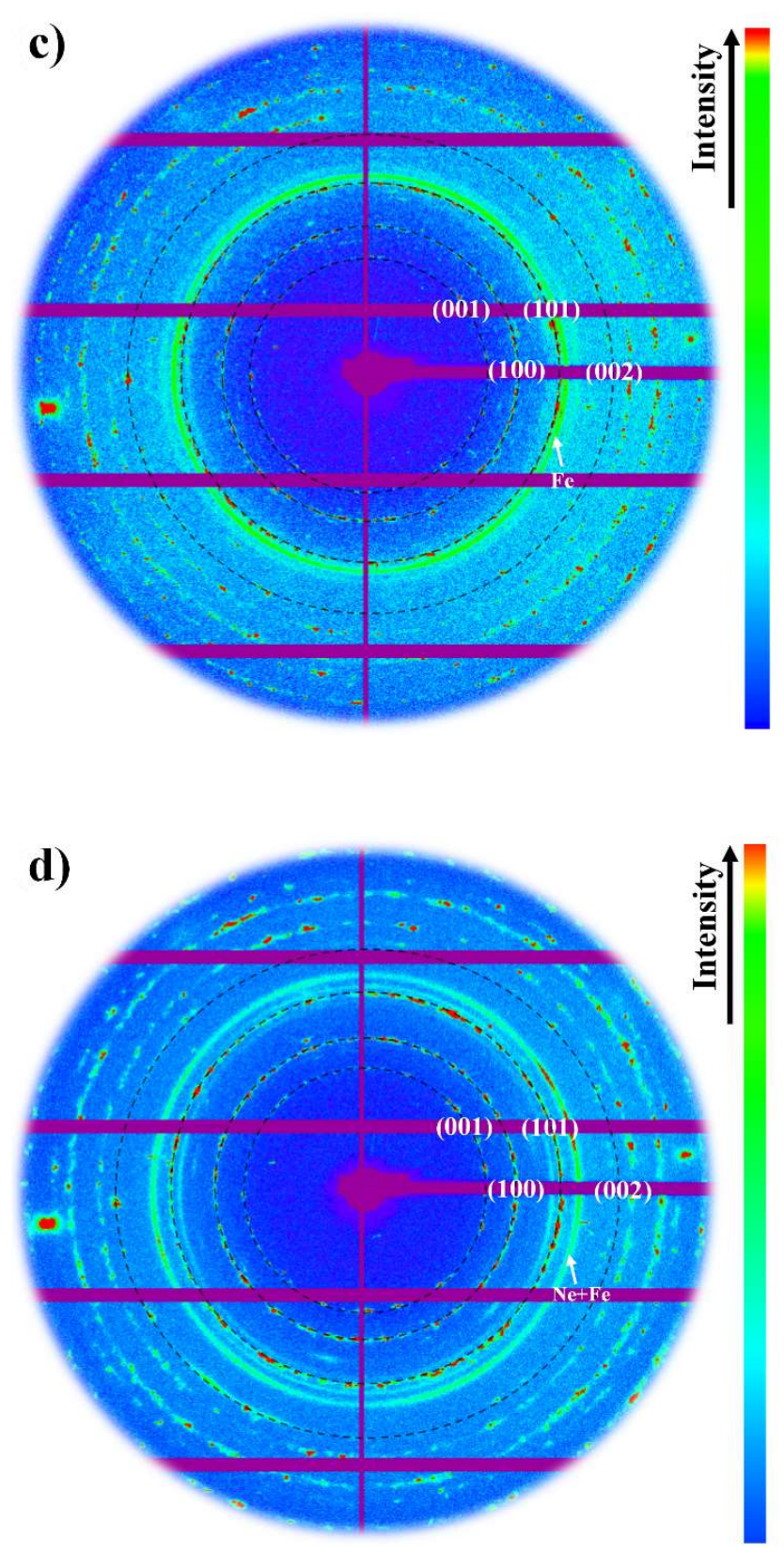

Figure S2. $(a-b)$ Representative high-pressure XRD images of the quinary HEB sample under compression at 9.8 GPa (a) and 52.7 GPa (b). (c-d) Representative high-pressure XRD images of the senary HEB sample under compression at $10.1 \mathrm{GPa}(\mathrm{c})$ and $55.8 \mathrm{GPa}$ (d). 

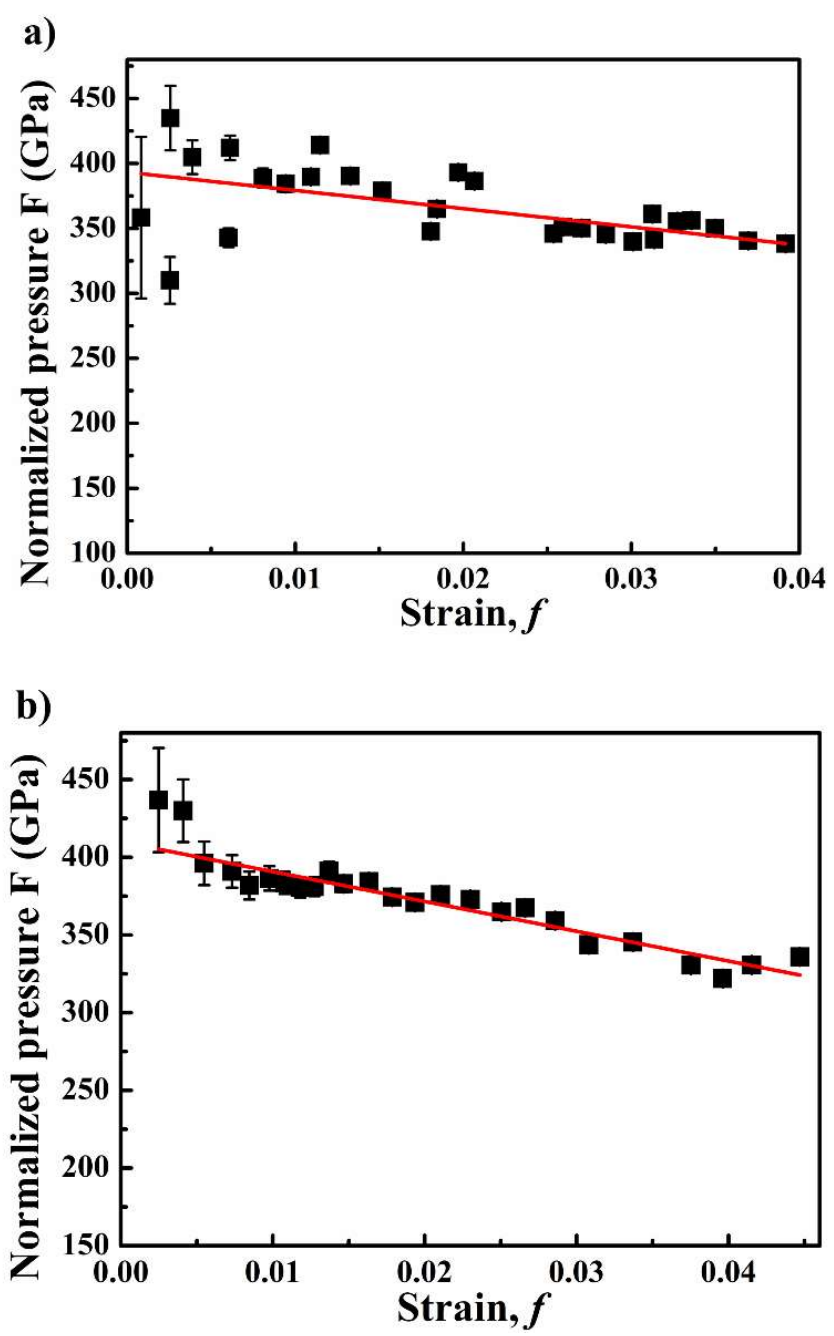

Figure S3. $(\mathrm{a}-\mathrm{b})$ The relationship between Normalized pressure $(\mathrm{F})$ and Eulerian strain $(f)$ of quinary (a) and the senary (b) HEBs. 

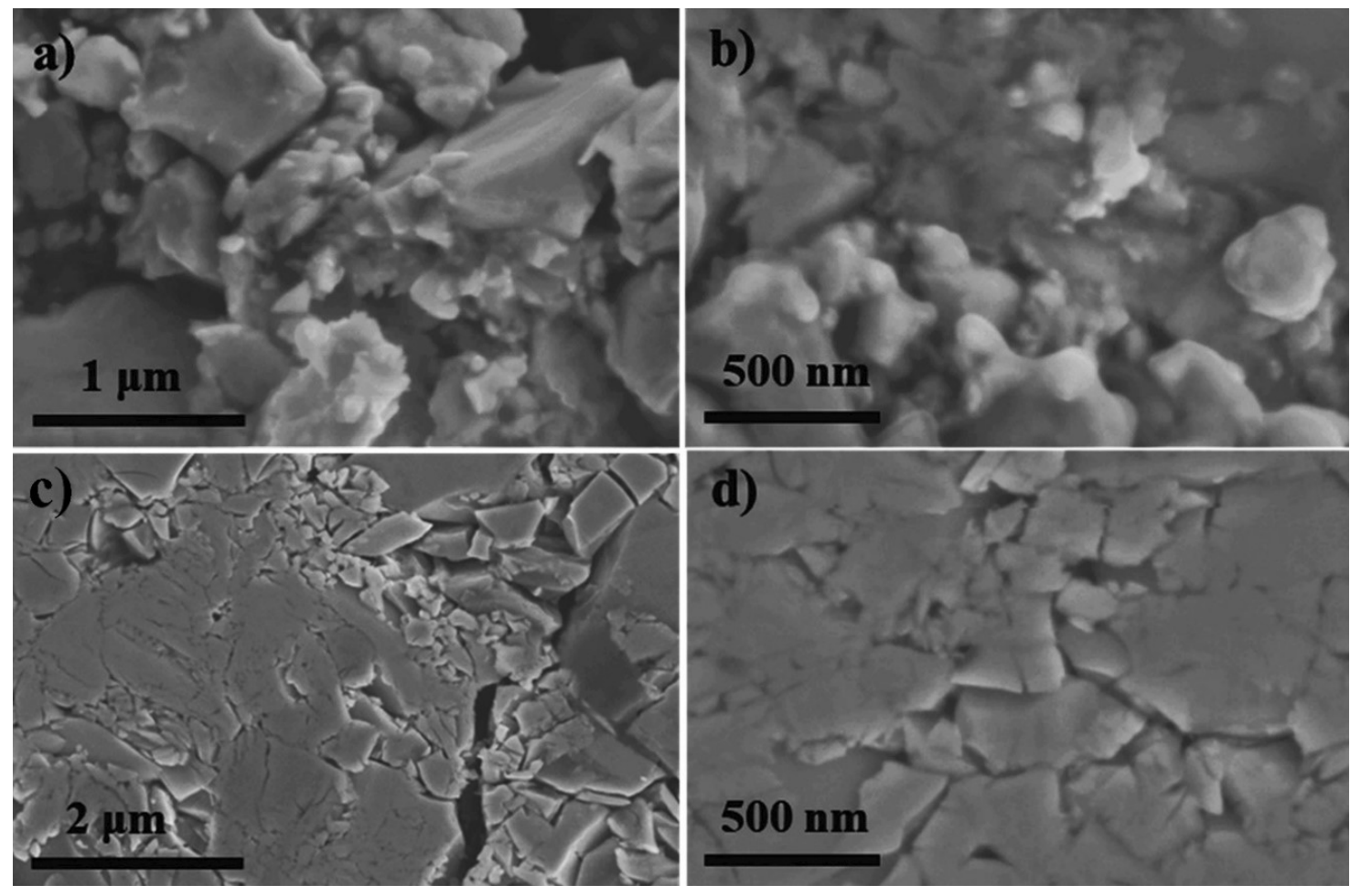

Figure S4. $(a-d)$ SEM images of the quinary $(a-b)$ and senary $(c-d)$ HEB samples quenched from $\sim 55 \mathrm{GPa}$. 

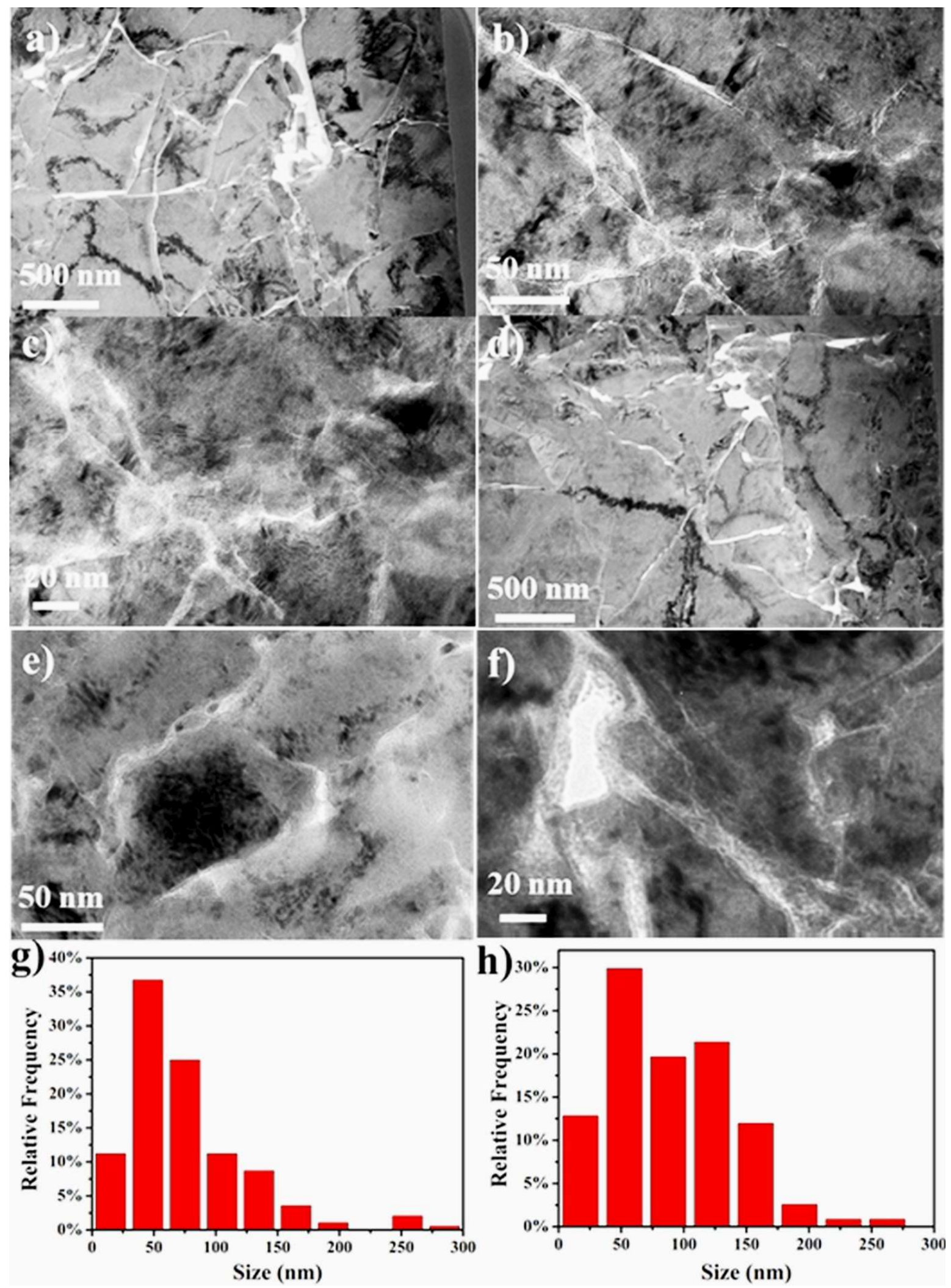
Figure S5. $(a-f)$ TEM images of the quinary $(a-c)$ and senary $(d-f)$ HEB samples quenched from $\sim 55 \mathrm{GPa}$. $(\mathrm{g}-\mathrm{f})$ Crystallite size distributions estimated from TEM images in the quenched quinary (g) and senary (h) HEBs.

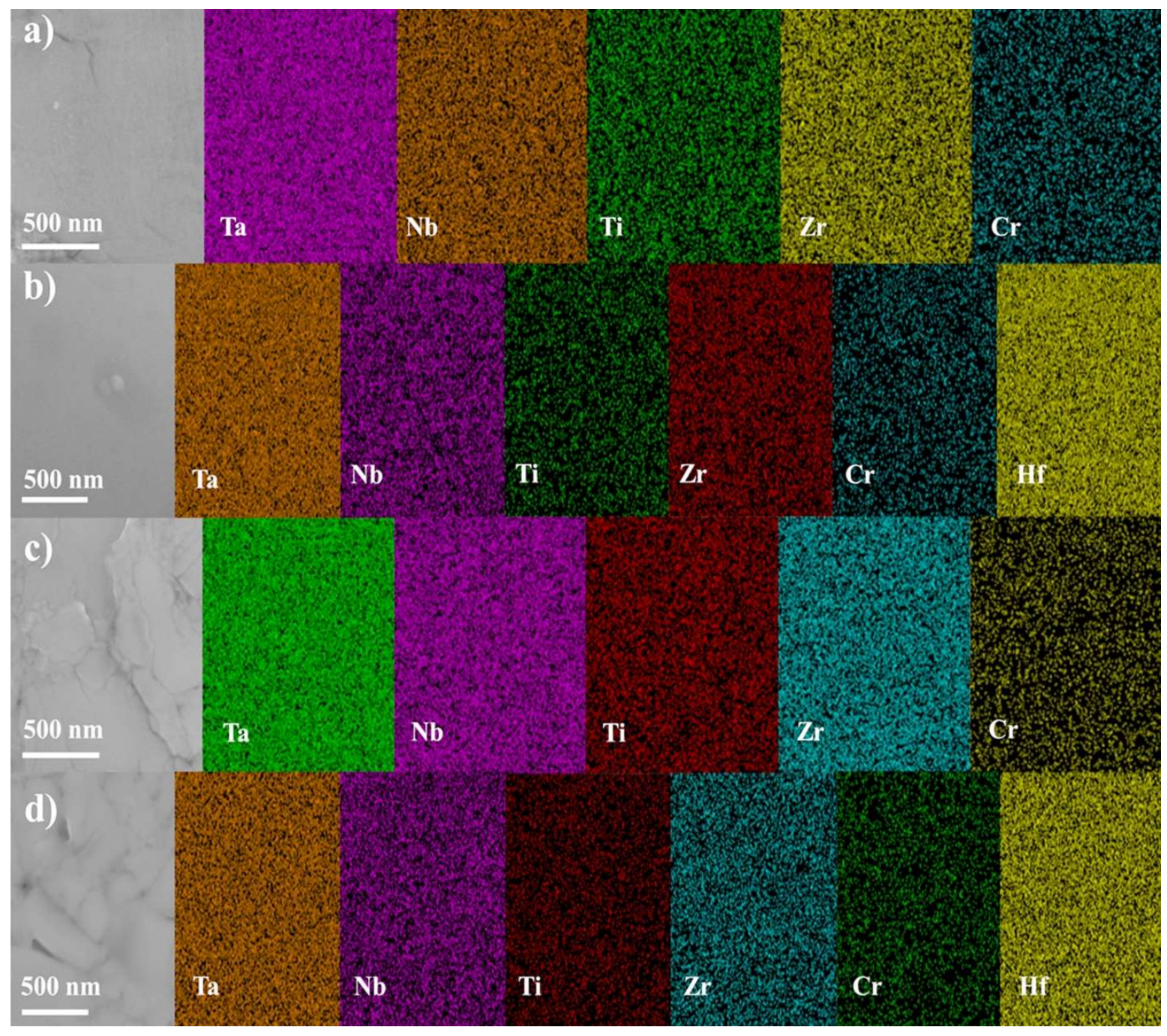

Figure S6. $(\mathrm{a}-\mathrm{d})$ Micron-scale EDS elemental mapping showing metal distributions in the quinary (a) and the senary (b) HEBs at ambient pressure, and in the quinary (c) and the senary (d) HEBs after quenching from compressions to $\sim 55 \mathrm{GPa}$. 

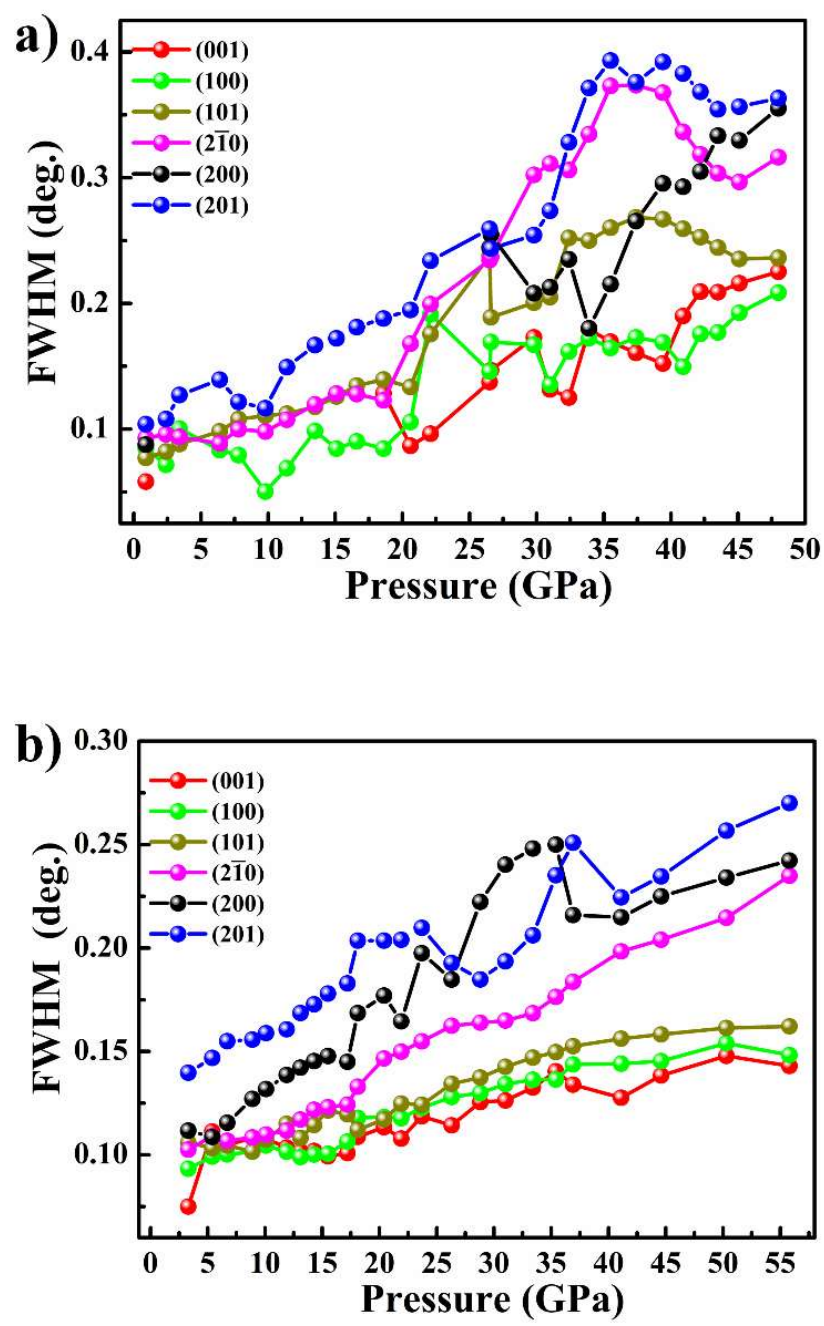

Figure S7. $(a-b)$ The full width at half maxima (FWHM) of XRD peaks of the quinary (a) and senary (b) HEBs at different pressures in compression. 
Table S1. Atomic percentages of transition metal elements in the synthesized quinary and senary HEB samples measured by EDS and XRF.

\begin{tabular}{|l|l|l|l|l|}
\hline element & quinary by EDS & quinary by XRF & senary by EDS & senary by XRF \\
\hline $\mathrm{Ti}$ & 21.4 & 21.0 & 16.9 & 17.9 \\
\hline $\mathrm{Cr}$ & 16.9 & 14.1 & 15.5 & 11.2 \\
\hline $\mathrm{Zr}$ & 17.4 & 18.4 & 15.8 & 15.3 \\
\hline $\mathrm{Nb}$ & 21.6 & 22.5 & 18.0 & 17.4 \\
\hline $\mathrm{Ta}$ & 22.7 & 24.0 & 15.6 & 20.4 \\
\hline $\mathrm{Hf}$ & $/$ & & 18.2 & 17.8 \\
\hline $\mathrm{Total}:$ & 100.0 & 100.0 & 100.0 & 100.0 \\
\hline
\end{tabular}


Table S2. Hardness and Young's moduli of simple and high-entropy diborides.

\begin{tabular}{|c|c|c|c|c|}
\hline Diboride & $\begin{array}{l}\text { Vickers hardness } \\
H v(\mathrm{GPa})\end{array}$ & $\begin{array}{l}\text { Nanoindentation } \\
\text { hardness } \\
H_{N I T}(\mathrm{GPa})\end{array}$ & $\begin{array}{l}\text { Young's } \\
\text { modulus } \\
E(\mathrm{GPa})\end{array}$ & $\begin{array}{l}\text { Refs. for } H v \& \\
E\end{array}$ \\
\hline $\mathrm{TaB}_{2}$ & 24.5 & $30.6^{a}$ & 497 & $11-12$ \\
\hline $\mathrm{NbB}_{2}$ & 25.5 & $31.9^{\mathrm{a}}$ & 508 & $11-12$ \\
\hline $\mathrm{ZrB}_{2}$ & $21.6-24.5$ & $27.0-30.6^{\mathrm{a}}$ & 523 & $11-12$ \\
\hline $\mathrm{CrB}_{2}$ & 20.6 & $25.8^{a}$ & 417 & $11-12$ \\
\hline $\mathrm{TiB}_{2}$ & $33.1-35.7$ & $41.4-44.6^{\mathrm{a}}$ & 583 & $11-12$ \\
\hline $\mathrm{HfB}_{2}$ & 28.4 & $35.5^{\mathrm{a}}$ & 535 & $11-12$ \\
\hline $\begin{array}{l}\text { quinary } \\
\text { HEB }\end{array}$ & $\begin{array}{l}27.3 \pm 3.8(1.0 \mathrm{~N})^{\mathrm{b}} \\
27.8 \pm 1.0(2.0 \mathrm{~N})^{\mathrm{b}} \\
25.2 \pm 0.6(2.9 \mathrm{~N})^{\mathrm{b}} \\
23.8 \pm 1.1(4.9 \mathrm{~N})^{\mathrm{b}} \\
22.0 \pm 2.7(9.8 \mathrm{~N})^{\mathrm{b}} \\
(25.6)^{\mathrm{c}}\end{array}$ & $\begin{array}{l}37.5 \pm 3.7 \\
(32.0)^{\mathrm{c}}\end{array}$ & $\begin{array}{l}549.2 \pm 43.9 \\
(505.6)^{\mathrm{c}}\end{array}$ & this work \\
\hline $\begin{array}{l}\text { senary } \\
\text { HEB }\end{array}$ & $\begin{array}{l}17.5 \pm 1.1(1.0 \mathrm{~N})^{\mathrm{b}} \\
24.3 \pm 1.9(2.0 \mathrm{~N})^{\mathrm{b}} \\
18.6 \pm 2.3(2.9 \mathrm{~N})^{\mathrm{b}} \\
(26.1)^{\mathrm{c}}\end{array}$ & $\begin{array}{l}32.2 \pm 5.8 \\
(32.6)^{\mathrm{c}}\end{array}$ & $\begin{array}{l}514.1 \pm 92.3 \\
(510.5)^{\mathrm{c}}\end{array}$ & this work \\
\hline
\end{tabular}

${ }^{\text {a }}$ Calculated using the approximate relation $H_{N I T}=1.25 H v^{13}$;

${ }^{\mathrm{b}}$ Loading in Vickers hardness test;

${ }^{\mathrm{c}}$ Averaged quantity of constituent simple diborides. 
Table S3. Young's modulus $(E)$ and nanoindentation hardness $\left(H_{N I T}\right)$ of the quinary HEB.

\begin{tabular}{|l|l|l|l|l|}
\hline Test \# & $\begin{array}{l}E \\
(\mathrm{GPa})\end{array}$ & $\begin{array}{l}\Delta E^{a} \\
(\mathrm{GPa})\end{array}$ & $\begin{array}{l}H_{N I T} \\
(\mathrm{GPa})\end{array}$ & $\begin{array}{l}\Delta H_{N I T}{ }^{a} \\
(\mathrm{GPa})\end{array}$ \\
\hline 1 & 489.5 & 7.7 & 41.0 & 1.0 \\
\hline 2 & 552.1 & 17.1 & 38.0 & 0.4 \\
\hline 3 & 561.8 & 16.8 & 35.9 & 0.6 \\
\hline 4 & 533.9 & 14.9 & 31.0 & 0.6 \\
\hline 5 & 538.5 & 9.7 & 41.1 & 0.5 \\
\hline 6 & 518.2 & 13.3 & 38.5 & 0.4 \\
\hline 7 & 633.5 & 16.7 & 41.9 & 0.8 \\
\hline 8 & 597.0 & 15.7 & 36.6 & 0.5 \\
\hline 9 & 518.1 & 8.8 & 33.2 & 0.4 \\
\hline $\begin{array}{l}\text { Mean of all } \\
\text { tests }\end{array}$ & 549.2 & & 37.5 & \\
\hline $\begin{array}{l}\text { Std. dev. of } \\
\text { all tests }\end{array}$ & 43.9 & & & \\
\hline
\end{tabular}

${ }^{a}$ standard deviation in a single test. 
Table S4. Young's modulus $(E)$ and nanoindentation hardness $\left(H_{N I T}\right)$ of the senary HEB.

\begin{tabular}{|l|l|l|l|l|}
\hline Test \# & $\begin{array}{l}E \\
(\mathrm{GPa})\end{array}$ & $\begin{array}{l}\Delta E^{a} \\
(\mathrm{GPa})\end{array}$ & $\begin{array}{l}H_{N I T} \\
(\mathrm{GPa})\end{array}$ & $\begin{array}{l}\Delta H_{N I T}{ }^{a} \\
(\mathrm{GPa})\end{array}$ \\
\hline 1 & 471.9 & 10.6 & 30.1 & 1.0 \\
\hline 2 & 634.3 & 14.7 & 40.9 & 0.9 \\
\hline 3 & 576.8 & 8.4 & 36.1 & 0.5 \\
\hline 4 & 556.5 & 16.9 & 34.4 & 0.4 \\
\hline 5 & 547.0 & 13.3 & 32.1 & 0.6 \\
\hline 6 & 455.1 & 10.5 & 28.7 & 0.5 \\
\hline 7 & 357.1 & 5.7 & 22.8 & 0.6 \\
\hline $\begin{array}{l}\text { Mean of all } \\
\text { tests }\end{array}$ & 514.1 & & 32.2 & \\
\hline $\begin{array}{l}\text { Std. dev. of } \\
\text { all tests }\end{array}$ & 92.3 & & & \\
\hline
\end{tabular}

${ }^{\mathrm{a}}$ standard deviation in a single test. 
Table S5. The cell parameters and unit cell volumes of the quinary HEB derived from HPXRD data.

\begin{tabular}{|c|c|c|c|c|c|c|}
\hline $\begin{array}{l}\text { Pressure } \\
(\mathrm{GPa})\end{array}$ & $\begin{array}{l}a \\
(\AA)\end{array}$ & $\begin{array}{l}\Delta a \\
(\AA)\end{array}$ & $\begin{array}{l}c \\
(\AA)\end{array}$ & $\begin{array}{l}\Delta \mathrm{c} \\
(\AA)\end{array}$ & $\begin{array}{l}V \\
\left(\AA^{3}\right)\end{array}$ & $\begin{array}{l}\Delta V \\
\left(\AA^{3}\right)\end{array}$ \\
\hline 0.9 & 3.0881 & 0.0007 & 3.2853 & 0.0011 & 27.13 & 0.02 \\
\hline 2.4 & 3.0724 & 0.0007 & 3.3021 & 0.0018 & 27.00 & 0.02 \\
\hline 3.4 & 3.0704 & 0.0006 & 3.3060 & 0.0009 & 26.99 & 0.02 \\
\hline 4.8 & 3.0721 & 0.0006 & 3.2895 & 0.0008 & 26.89 & 0.01 \\
\hline 6.4 & 3.0601 & 0.0006 & 3.2942 & 0.0009 & 26.71 & 0.01 \\
\hline 7.8 & 3.0627 & 0.0005 & 3.2878 & 0.0010 & 26.70 & 0.01 \\
\hline 9.8 & 3.0505 & 0.0007 & 3.2952 & 0.0018 & 26.55 & 0.01 \\
\hline 11.4 & 3.0472 & 0.0007 & 3.2889 & 0.0018 & 26.45 & 0.01 \\
\hline 13.5 & 3.0419 & 0.0007 & 3.2858 & 0.0018 & 26.33 & 0.01 \\
\hline 15.1 & 3.0379 & 0.0007 & 3.2894 & 0.0018 & 26.29 & 0.01 \\
\hline 16.6 & 3.0338 & 0.0007 & 3.2810 & 0.0018 & 26.15 & 0.02 \\
\hline 18.6 & 3.0320 & 0.0007 & 3.2623 & 0.0018 & 26.01 & 0.01 \\
\hline 20.6 & 3.0267 & 0.0006 & 3.2508 & 0.0014 & 25.79 & 0.01 \\
\hline 22.1 & 3.0237 & 0.0006 & 3.2536 & 0.0014 & 25.76 & 0.01 \\
\hline 25.6 & 3.0218 & 0.0005 & 3.2458 & 0.0018 & 25.67 & 0.01 \\
\hline 26.5 & 3.0163 & 0.0006 & 3.2488 & 0.0014 & 25.60 & 0.02 \\
\hline 29.8 & 3.0022 & 0.0006 & 3.2353 & 0.0014 & 25.25 & 0.01 \\
\hline 31.0 & 3.0015 & 0.0006 & 3.2316 & 0.0014 & 25.21 & 0.01 \\
\hline
\end{tabular}




\begin{tabular}{|l|l|l|l|l|l|l|}
\hline 32.4 & 3.001 & 0.0006 & 3.2230 & 0.0013 & 25.13 & 0.01 \\
\hline 33.9 & 2.9978 & 0.0006 & 3.2162 & 0.0013 & 25.03 & 0.01 \\
\hline 35.5 & 2.9942 & 0.0006 & 3.2092 & 0.0013 & 24.92 & 0.01 \\
\hline 37.4 & 2.9888 & 0.0006 & 3.2093 & 0.0013 & 24.83 & 0.01 \\
\hline 39.4 & 2.9869 & 0.0006 & 3.2143 & 0.0009 & 24.83 & 0.02 \\
\hline 40.9 & 2.9837 & 0.0006 & 3.2080 & 0.0009 & 24.73 & 0.01 \\
\hline 42.2 & 2.9814 & 0.0006 & 3.2052 & 0.0009 & 24.67 & 0.01 \\
\hline 43.5 & 2.9742 & 0.0006 & 3.2081 & 0.0009 & 24.58 & 0.02 \\
\hline 45.1 & 2.9700 & 0.0006 & 3.1996 & 0.0009 & 24.44 & 0.01 \\
\hline 48.0 & 2.9600 & 0.0005 & 3.1910 & 0.0009 & 24.29 & 0.01 \\
\hline 52.7 & 2.9525 & 0.0006 & 3.1782 & 0.0010 & 23.99 & 0.01 \\
\hline $\mathrm{D}^{\mathrm{a}} 44.1$ & 2.9752 & 0.0006 & 3.2115 & 0.0009 & 24.62 & 0.01 \\
\hline $\mathrm{D}^{\mathrm{a}} 42.5$ & 2.9668 & 0.0006 & 3.2080 & 0.0009 & 24.45 & 0.01 \\
\hline $\mathrm{D}^{\mathrm{a}} 34.6$ & 2.9898 & 0.0006 & 3.2329 & 0.0009 & 25.03 & 0.01 \\
\hline $\mathrm{D}^{\mathrm{a}} 28.1$ & 3.0034 & 0.0006 & 3.2496 & 0.0009 & 25.39 & 0.01 \\
\hline $\mathrm{D}^{\mathrm{a}} 22.0$ & 3.0300 & 0.0006 & 3.2706 & 0.0010 & 26.00 & 0.02 \\
\hline $\mathrm{D}^{\mathrm{a}} 9.4$ & 3.0659 & 0.0006 & 3.2993 & 0.0010 & 26.86 & 0.02 \\
\hline $\mathrm{D}^{\mathrm{a}} 3.8$ & 3.0780 & 0.0006 & 3.3058 & 0.0010 & 27.12 & 0.02 \\
\hline $\mathrm{D}^{\mathrm{a}} 0.3$ & 3.0794 & 0.0006 & 3.3073 & 0.0010 & 27.16 & 0.02 \\
\hline
\end{tabular}

a "D" denotes decompression. 
Table S6. The cell parameters and unit cell volumes of the senary HEB derived from HPXRD data.

\begin{tabular}{|l|l|l|l|l|l|l|}
\hline $\begin{array}{l}\text { Pressure } \\
(\mathrm{GPa})\end{array}$ & $\begin{array}{l}a \\
(\AA)\end{array}$ & $\begin{array}{l}\Delta a \\
(\AA)\end{array}$ & $\left.\begin{array}{l}c \\
\AA\end{array}\right)$ & $\begin{array}{l}\Delta \mathrm{c} \\
(\AA)\end{array}$ & $\begin{array}{l}V \\
\left(\AA^{3}\right)\end{array}$ & $\begin{array}{l}\Delta V \\
\left(\AA^{3}\right)\end{array}$ \\
\hline 3.3 & 3.0826 & 0.0005 & 3.3290 & 0.0019 & 27.40 & 0.02 \\
\hline 5.4 & 3.0771 & 0.0005 & 3.3250 & 0.0019 & 27.26 & 0.02 \\
\hline 6.7 & 3.0737 & 0.0005 & 3.3185 & 0.0019 & 27.15 & 0.02 \\
\hline 8.9 & 3.0677 & 0.0005 & 3.3134 & 0.0019 & 27.00 & 0.02 \\
\hline 10.1 & 3.0650 & 0.0005 & 3.3083 & 0.0019 & 26.91 & 0.02 \\
\hline 11.9 & 3.0611 & 0.0005 & 3.3037 & 0.0019 & 26.81 & 0.02 \\
\hline 13.1 & 3.0583 & 0.0005 & 3.3000 & 0.0019 & 26.73 & 0.02 \\
\hline 14.3 & 3.0557 & 0.0005 & 3.2958 & 0.0019 & 26.65 & 0.02 \\
\hline 15.5 & 3.0527 & 0.0005 & 3.2933 & 0.0019 & 26.59 & 0.02 \\
\hline 17.2 & 3.0499 & 0.0005 & 3.2900 & 0.0019 & 26.50 & 0.01 \\
\hline 18.1 & 3.0471 & 0.0005 & 3.2870 & 0.0019 & 26.43 & 0.01 \\
\hline 20.4 & 3.0428 & 0.0005 & 3.2803 & 0.0019 & 26.30 & 0.01 \\
\hline 21.9 & 3.0387 & 0.0005 & 3.2745 & 0.0019 & 26.18 & 0.01 \\
\hline 23.7 & 3.0350 & 0.0005 & 3.2681 & 0.0019 & 26.07 & 0.01 \\
\hline 26.3 & 3.0308 & 0.0005 & 3.2614 & 0.0018 & 25.95 & 0.02 \\
\hline 28.8 & 3.0261 & 0.0005 & 3.2532 & 0.0018 & 25.80 & 0.01 \\
\hline 31.0 & 3.0211 & 0.0005 & 3.2449 & 0.0018 & 25.65 & 0.01 \\
\hline
\end{tabular}




\begin{tabular}{|l|l|l|l|l|l|l|}
\hline 33.4 & 3.0169 & 0.0005 & 3.2395 & 0.0018 & 25.53 & 0.01 \\
\hline 35.4 & 3.0120 & 0.0005 & 3.2318 & 0.0018 & 25.39 & 0.01 \\
\hline 36.9 & 3.0062 & 0.0005 & 3.2240 & 0.0018 & 25.23 & 0.01 \\
\hline 41.1 & 2.9958 & 0.0005 & 3.2200 & 0.0018 & 25.03 & 0.01 \\
\hline 44.6 & 2.9863 & 0.0005 & 3.2061 & 0.0018 & 24.76 & 0.01 \\
\hline 46.3 & 2.9808 & 0.0005 & 3.1993 & 0.0018 & 24.62 & 0.01 \\
\hline 50.3 & 2.9757 & 0.0005 & 3.1932 & 0.0018 & 24.49 & 0.01 \\
\hline 55.8 & 2.9666 & 0.0005 & 3.1848 & 0.0017 & 24.27 & 0.01 \\
\hline $\mathrm{D}^{\mathrm{a}} 51.8$ & 2.9710 & 0.0005 & 3.1873 & 0.0017 & 24.36 & 0.01 \\
\hline $\mathrm{D}^{\mathrm{a}} 48.6$ & 2.9764 & 0.0005 & 3.1945 & 0.0018 & 24.51 & 0.01 \\
\hline $\mathrm{D}^{\mathrm{a}} 45.7$ & 2.9863 & 0.0005 & 3.2096 & 0.0018 & 24.79 & 0.01 \\
\hline $\mathrm{D}^{\mathrm{a}} 44.3$ & 2.9919 & 0.0005 & 3.2164 & 0.0018 & 24.93 & 0.01 \\
\hline $\mathrm{D}^{\mathrm{a}} 40.4$ & 3.0007 & 0.0005 & 3.2232 & 0.0018 & 25.13 & 0.01 \\
\hline $\mathrm{D}^{\mathrm{a}} 36.4$ & 3.0105 & 0.0005 & 3.2366 & 0.0018 & 25.40 & 0.01 \\
\hline $\mathrm{D}^{\mathrm{a}} 32.0$ & 3.0198 & 0.0005 & 3.2513 & 0.0018 & 25.68 & 0.01 \\
\hline $\mathrm{D}^{\mathrm{a}} 27.8$ & 3.0301 & 0.0005 & 3.2633 & 0.0018 & 25.95 & 0.01 \\
\hline $\mathrm{D}^{\mathrm{a}} 23.6$ & 3.0405 & 0.0005 & 3.2752 & 0.0018 & 26.22 & 0.01 \\
\hline $\mathrm{D}^{\mathrm{a}} 19.1$ & 3.0485 & 0.0005 & 3.2887 & 0.0018 & 26.47 & 0.01 \\
\hline $\mathrm{D}^{\mathrm{a}} 15.0$ & 3.0582 & 0.0005 & 3.3031 & 0.0018 & 26.75 & 0.02 \\
\hline $\mathrm{D}^{\mathrm{a}} 4.3$ & 3.0791 & 0.0005 & 3.3369 & 0.0018 & 27.40 & 0.02 \\
\hline $\mathrm{D}^{\mathrm{a}} 1.8$ & 3.0871 & 0.0005 & 3.3449 & 0.0018 & 27.61 & 0.02 \\
\hline $\mathrm{D}^{\mathrm{a}} 0$ & 3.0926 & 0.0005 & 3.3494 & 0.0019 & 27.74 & 0.02 \\
\hline
\end{tabular}

a "D" denotes decompression. 
Table S7. Comparisons between the bulk moduli ( $B_{0}$ in GPa) of simple or high-entropy diborides derived from DFT calculations and those obtained from experimental determinations (EOS fitting at $B_{0}{ }^{\prime}=4$ except as indicated).

\begin{tabular}{|c|c|c|c|c|c|c|c|c|c|c|c|c|c|}
\hline $\begin{array}{l}\text { DFT } \\
\text { method }\end{array}$ & $\mathrm{TiB}_{2}$ & $\mathrm{CrB}_{2}$ & $\mathrm{ZrB}_{2}$ & $\mathrm{NbB}_{2}$ & $\mathrm{MoB}_{2}$ & $\mathrm{HfB}_{2}$ & $\mathrm{TaB}_{2}$ & $\mathrm{WB}_{2}$ & $\mathrm{OsB}_{2}$ & $\mathrm{ReB}_{2}$ & $\mathrm{RMS}^{\mathrm{f}}$ & $\begin{array}{l}\text { quinary } \\
\text { HEB }\end{array}$ & $\begin{array}{l}\text { senary } \\
\text { HEB }\end{array}$ \\
\hline CASTEP $^{\text {a }}$ & $\begin{array}{l}248 \\
(3.9)\end{array}$ & $\begin{array}{l}274 \\
(5.4)\end{array}$ & $\begin{array}{l}238 \\
(3.9)\end{array}$ & $\begin{array}{l}284 \\
(4.0)\end{array}$ & $\begin{array}{l}295 \\
(4.3)\end{array}$ & $\begin{array}{l}270 \\
(2.5)\end{array}$ & $\begin{array}{l}297 \\
(4.1)\end{array}$ & $\begin{array}{l}305 \\
(4.7)\end{array}$ & & & 22.1 & $\begin{array}{l}261 \\
(4.1)\end{array}$ & $\begin{array}{l}248 \\
(4.7)\end{array}$ \\
\hline CASTEP $^{b}$ & 243 & 259 & 233 & 278 & 293 & 290 & 318 & 327 & 317 & 327 & 23.8 & 260 & 266 \\
\hline $\mathrm{CP} 2 \mathrm{~K}^{\mathrm{c}}$ & 252 & 225 & 243 & 279 & 305 & 253 & 290 & 302 & & & 19.9 & 249 & 248 \\
\hline $\mathrm{QE}^{\mathrm{d}}$ & 250 & 293 & 237 & 288 & 309 & 253 & 302 & 332 & 315 & 337 & 26.8 & 257 & 253 \\
\hline CASTEP $^{\mathrm{e}}$ & 253 & 289 & 238 & 287 & 330 & 261 & 296 & 325 & & & 25.0 & & \\
\hline $\begin{array}{l}\text { Materials } \\
\text { Project } 14\end{array}$ & 253 & 252 & 238 & 287 & 299 & 251 & 302 & 295 & 311 & 337 & 26.5 & & \\
\hline AFLOW $^{15}$ & 244 & 188 & 238 & & & 251 & 295 & & & 332 & 24.7 & & \\
\hline $\begin{array}{l}\text { Ave. calc. } \\
B_{0}\end{array}$ & 249 & 254 & 238 & 284 & 305 & 261 & 300 & 314 & 314 & 333 & 23.1 & 257 & 254 \\
\hline $\begin{array}{l}\text { Expt. } \\
\text { Refs. }{ }^{16-23}\end{array}$ & $\begin{array}{l}250 \\
16 \\
260 \\
17\end{array}$ & $\begin{array}{l}240 \\
16 \\
230 \\
17\end{array}$ & $\begin{array}{l}240 \\
16\end{array}$ & & $\begin{array}{l}296 \\
18\end{array}$ & $\begin{array}{l}270 \\
19\end{array}$ & $\begin{array}{l}335 \\
20\end{array}$ & $\begin{array}{l}349 \\
18\end{array}$ & $\begin{array}{l}395 \\
(1.4) \\
21 \\
365 \\
21\end{array}$ & $\begin{array}{l}364 \\
22 \\
360 \\
23\end{array}$ & & $\begin{array}{l}393 * \\
(1.6) \\
337 *\end{array}$ & $\begin{array}{l}404 * \\
(1.1) \\
318 *\end{array}$ \\
\hline Expt. Ref. ${ }^{24}$ & $\begin{array}{l}252 \\
256 \\
240\end{array}$ & & $\begin{array}{l}231 \\
232 \\
248 \\
240\end{array}$ & 287 & & $\begin{array}{l}256 \\
242 \\
259 \\
263 \\
269\end{array}$ & 294 & & & & & & \\
\hline $\begin{array}{l}\text { Ave. expt. } \\
B_{0}\end{array}$ & 252 & 235 & 238 & 287 & 296 & 260 & 315 & 349 & $\begin{array}{l}395 \\
(1.4) \\
365\end{array}$ & 362 & & $\begin{array}{l}393 \\
(1.6) \\
337\end{array}$ & $\begin{array}{l}404 \\
(1.1) \\
318\end{array}$ \\
\hline
\end{tabular}

${ }^{a}$ CASTEP (Academic V.19.11) with program-generated norm-conserving pseudopotentials (NCP). In EOS fitting, $B_{0}^{\prime}$ was fitted (as indicated in the parenthesis).

${ }^{\mathrm{b}}$ CASTEP (Academic V.19.11) with NCP generated from the OPIUM ${ }^{25}$ program with the atomic parameters taken from

https://www.sas.upenn.edu/rappegroup/research/pseudo-potential-gga.html.

${ }^{\mathrm{c}} \mathrm{CP} 2 \mathrm{~K}$ V.4.0.

${ }^{\mathrm{d}}$ Quantum ESPRESSO V.6.1 with standard solid-state pseudopotentials (SSSP) for precision (https://www.materialscloud.org/discover/sssp/table/efficiency). 
${ }^{\text {e }}$ CASTEP with ultra-soft pseudopotentials (USP) ${ }^{12}$.

${ }^{\mathrm{f}}$ Root-mean-square of the deviations between the DFT calculated $B_{0}$ and the averaged experimental values of the simple diborides.

* This work. 


\section{REFERENCES}

(1) The GIMP Development Team, GNU Image Manipulation Program (V. 2.10), https://www.gimp.org/, accessed: December, 2020.

(2) Brazhkin, V. V.; Solozhenko, V. L. Myths about new ultrahard phases: Why materials that are significantly superior to diamond in elastic moduli and hardness are impossible. $J$. Appl. Phys. 2019, 125, 130901.

(3) Prescher, C.; Prakapenka, V. B. DIOPTAS: a program for reduction of two-dimensional X-ray diffraction data and data exploration. High Pressure Res. 2015, 35, 223-230.

(4) Clark, S. J.; Segall, M. D.; Pickard, C. J.; Hasnip, P. J.; Probert, M. I.; Refson, K.; Payne, M. C. First principles methods using CASTEP. Z. Kristallogr. 2005, 220, 567-570.

(5) Kühne, T. D.; Iannuzzi, M.; Ben, D. M.; Rybkin, V. V.; Seewald, P.; Stein, F.; Laino, T.; Khaliullin, R. Z.; Schütt, O.; Schiffmann, F.; et. al. CP2K: An electronic structure and molecular dynamics software package-Quickstep: Efficient and accurate electronic structure calculations. J. Chem. Phys. 2020, 152, 194103.

(6) Giannozzi, P.; Baroni, S.; Bonini, N.; Calandra, M.; Car, R.; Cavazzoni, C.; Ceresoli, D.; Chiarotti, G. L.; Cococcioni, M.; Dabo, I.; et. al. QUANTUM ESPRESSO: a modular and open-source software project for quantum simulations of materials. J. Phys.: Condens. Matter 2009, 21, 395502.

(7) Perdew, J. P.; Burke, K.; Ernzerhof, M. Generalized gradient approximation made simple. Phys. Rev. Lett. 1996, 77, 3865.

(8) Goedecker, S.; Teter, M.; Hutter, J. Separable dual-space Gaussian pseudopotentials. Phys. Rev. B 1996, 54, 1703.

(9) VandeVondele, J.; Hutter, J. Gaussian basis sets for accurate calculations on molecular systems in gas and condensed phases. J. Chem. Phys. 2007, 127, 114105.

(10) Gianluca, P.; M. Antimo.; E. C. Ivano.; M. Nicolas.; M. Nicola, A standard solid state pseudopotentials (SSSP) library optimized for precision and efficiency, Materials Cloud Archive, 2020. doi: 10.24435/materialscloud:2018.0001/v4. 
(11) Akopov, G.; Yeung, M. T.; Kaner, R. B. Rediscovering the crystal chemistry of borides. Adv. Mater. 2017, 29, 1604506.

(12)Zhou, Y.; Xiang, H.; Feng, Z.; Li, Z. General trends in electronic structure, stability, chemical bonding and mechanical properties of ultrahigh temperature ceramics $\mathrm{TMB}_{2}$ (TM= transition metal). J. Mater. Sci. Technol. 2015, 31, 285-294.

(13) Sawa, T. presented at IMEKO TC3, TC5, and TC22 Conf. Metrology in Modern Context, Correlation between nanoindentation test result and Vickers hardness, Thailand, Nov., 2010.

(14) Jain, A.; Ong, S. P.; Hautier, G.; Chen, W.; Richards, W. D.; Dacek, S.; Cholia, S.; Gunter, D.; Skinner, D.; Ceder, G. Commentary: The Materials Project: A materials genome approach to accelerating materials innovation. APL Mater. 2013, 1, 011002.

(15)Andreoni,W.; Yip, S. Handbook of Materials Modeling Methods: Theory and Modeling, Springer, Lausanne, Switzerland, 2020.

(16) Okamoto, N. L.; Kusakari, M.; Tanaka, K.; Inui, H.; Otani, S. Anisotropic elastic constants and thermal expansivities in monocrystal $\mathrm{CrB}_{2}, \mathrm{TiB}_{2}$, and $\mathrm{ZrB}_{2}$. Acta Mater. 2010, $58,76-84$.

(17)Waśkowska, A.; Gerward, L.; Olsen, J. S.; Babu, K. R.; Vaitheeswaran, G.; Kanchana, V.; Svane, A.; Filipov, V.; Levchenko, G.; Lyaschenko, A. Thermoelastic properties of $\mathrm{ScB}_{2}, \mathrm{TiB}_{2}, \mathrm{YB}_{4}$ and $\mathrm{HoB}_{4}$ : Experimental and theoretical studies. Acta Mater. 2011, 59, 4886-4894.

(18) Yin, S.; He, D.; Xu, C.; Wang, W.; Wang, H.; Li, L.; Zhang, L.; Liu, F.; Liu, P.; Wang, Z. Hardness and elastic moduli of high pressure synthesized $\mathrm{MoB}_{2}$ and $\mathrm{WB}_{2}$ compacts. High Pressure Res. 2013, 33, 409-417.

(19)Liang, H.; Peng, F.; Guan, S.; Tan, L.; Chen, H.; Lei, L.; He, D.; Lu, C. Abnormal physical behaviors of hafnium diboride under high pressure. Appl. Phys. Lett. 2019, 115, 231903.

(20) Winkler, B.; Juarez-Arellano, E. A.; Friedrich, A.; Bayarjargal, L.; Schröder, F.; 
Biehler, J.; Milman, V.; Clark, S. M.; Yan, J. In situ synchrotron X-ray diffraction study of the formation of $\mathrm{TaB}_{2}$ from the elements in a laser heated diamond anvil cell. Solid State Sci. 2010, 12, 2059-2064.

(21)Cumberland, R. W.; Weinberger, M. B.; Gilman, J. J.; Clark, S. M.; Tolbert, S. H.; Kaner, R. B. Osmium diboride, an ultra-incompressible, hard material. J. Am. Chem. Soc. 2005, 127, 7264-7265.

(22)Burrage, K. C.; Lin, C. M.; Chen, W. C.; Chen, C. C.; Vohra, Y. K. Experimental and Computational Studies on Superhard Material Rhenium Diboride under Ultrahigh Pressures. Materials 2020, 13, 1657.

(23) Chung, H. Y.; Weinberger, M. B.; Levine, J. B.; Kavner, A.; Yang, J. M.; Tolbert, S. H.; Kaner, R. B. Synthesis of Ultra-Incompressible Superhard Rhenium Diboride at Ambient Pressure. Science 2007, 316, 436-439.

(24)Fahrenholtz, W. G.; Wuchina, E. J.; Lee, W. E.; Zhou, Y., Ultra-high temperature ceramics: materials for extreme environment applications. Wiley, Hoboken, NJ, USA 2014. (25) Opium - pseudopotential generation project, V4.1. http://opium.sourceforge.net/. 